\title{
A INFLUÊNCIA DA OBESIDADE NO PROGNÓSTICO DA FERTILIZAÇÃO IN VITRO
}

\author{
THE INFLUENCE OF OBESITY IN THE PROGNOSIS OF IN VITRO FERTILIZATION
}

\begin{abstract}
Vinicius Medina Lopes, Anna Luiza Moraes Souza, Murilo Cezar Souza Oliveira, Pamela do Carmo Mesquita Pereira, Amanda Evelyn Cruvinel Goulart, Natalia Ivet Zavattiero Tierno, Thaisa Sant'Anna Lacerda e Jean Pierre Barguil Brasileiro
\end{abstract}

DOI - 10.5935/2236-5117.2015v52n3/4a02

\section{RESUMO}

Objetivo. Avaliar o impacto do índice de massa corpórea das pacientes submetidas a fertilização in vitro sobre as taxas de gravidez e de abortamento, em um centro privado de reprodução assistida em Brasília-DF, Brasil, no período de janeiro de 2014 a dezembro de 2016.

Método. Estudo retrospectivo em que foram analisados os resultados das transferências de embriões a fresco em pacientes submetidas a fertilização in vitro. Para análise estatística foi utilizado o teste exato de Fisher.

Resultados. Foram avaliados 567 ciclos de fertilização in vitro com transferências de embrião a fresco. A média do índice de massa corpórea foi 23,4. 0 total de 410 pacientes obtiveram índices de massa corpórea abaixo de 25 (grupo A), 135 apresentaram índice de 25 a 29,99 (grupo B) e 22 tiveram índice igual ou acima de 30 (grupo C). As taxas de gestação de cada grupo foram, respectivamente, para os grupos $A$, B e C, $54,8 \%$ (225 de 410 ), $45,9 \%$ (62 de135) e $40,9 \%$ (9 de 22 ), e as de abortamento foram as seguintes, nesta ordem: $13,8 \%$ (31 de 225), 17,8\% (11 de 62) e 66,6\% (6 de 9). As taxas de gestação em curso foram de $47,3 \%$ para o grupo $A$, $37,7 \%$ para o B e $13,6 \%$ para o C. 0 teste exato de Fisher resultou em diferenças significativas nas taxas de gestação evolutiva entre os grupos $A$ e $C(p<0,001)$ e entre B e C $(p<0,005)$.

Conclusão. Demonstrou-se que a obesidade feminina afeta negativamente a taxa de gestação e eleva as taxas de abortamento em pacientes submetidas a fertilização in vitro.

Palavras-chave. Obesidade; peso corporal; fertilização in vitro; taxa de gestação; taxa de abortamento

\section{ABSTRACT}

Objective. To evaluate the impact of the body mass index on pregnancy and miscarriage rates of patients undergoing in vitro fertilization in a private assisted reproduction center in
Vinicius Medina Lopes - médico, ginecologista e obstetra, mestre pela Universidade de Brasília, Instituto VERHUM, Brasília, Distrito Federal

Anna Luiza Moraes Souza - médica, ginecologista e obstetra, mestre pela universidade Federal de Minas Gerais, Instituto VERHUM

Murilo Cezar Souza Oliveira - médico, ginecologista e obstetra, Instituto VERHUM

Pamela do Carmo Mesquita Pereira - médica, ginecologista e obstetra, Instituto VERHUM

Amanda Evelyn Cruvinel Goulart - médica, ginecologista e obstetra, Instituto VERHUM

Natalia Ivet Zavattiero Tierno - médica, ginecologista e obstetra, Instituto VERHUM

Thaisa Sant'Anna Lacerda - bióloga, doutora pela Universidade de Brasília, Instituto VERHUM

Jean Pierre Barguil Brasileiro - médico, ginecologista e obstetra, Instituto VERHUM

Correspondência: Vinicius Medina Lopes. Instituto VERHUM, SHIS QI 3, Ed. Medical Plaza, sala n. ${ }^{\circ} 101$, CEP 701605-200, Brasília-DF.

Telefone: (61) 33654545, fax: (61) 33654543.

Internet: vinicius@verhum.com.br

Brazil from January 2014 to December 2016.

Method. Retrospective single institution study which analysed the results of fresh embryo transfer in patients undergoing in vitro fertilization at an assisted reproduction center in Brasilia-DF. Statistical analysis was performed by using Fisher's exact test. Units of measurement for body mass index are $\mathrm{kg} / \mathrm{m}^{2}$.

Results. There were 567 in vitro fertilization cycles with 
fresh embryo transfer evaluated. The median body mass index in this group was 23.4. A total of 410 patients had body mass index under 25 (group A), 135 patients had body mass index from 25 to 29.99 (group B) and 22 had body mass index equal or above 30 (group C). Pregnancy rates were, respectively, for groups $A, B$ and C, 54.8\% (225 from 410), 45.9\% (62 from 135) and 40.9\% (9 from 22$)$, miscarriage rates for the respective groups were $13.8 \%$ (31 from 225), 17.8\% (11 from 62) and 66.6\% (6 from 9). Ongoing pregnancy rates were $47.3 \%$ for group A, $37.7 \%$ for $B$ and $13.6 \%$ for $C$. Fisher's exact test resulted in significant differences between ongoing pregnancy rates on groups $A$ and $C(p<0.001)$ and between groups $B$ and $C(p<0.005)$.

Conclusion. Female obesity has been shown to negatively affect pregnancy rates and increases miscarriage rates in patients undergoing in vitro fertilization.

Key words. Obesity; body mass index; in vitro fertilization; pregnancy rate; miscarriage rate

\section{INTRODUÇÃO}

A obesidade é definida como excesso de gordura corporal. Tem causa multifatorial e constitui um grande problema de agravo à saúde global. Atualmente, cerca de metade da população adulta brasileira encontra-se obesa ou com sobrepeso. Nas Regiões Sul e Sudeste do País, a prevalência desses distúrbios é maior que $50 \% .{ }^{1}$ A taxa da obesidade nos países nórdicos varia de $18 \%$ a $28,8 \%$ na população feminina ${ }^{2}$ e, nos Estados Unidos, a taxa é de $39,8 \%$ nos indivíduos adultos. ${ }^{3} \mathrm{~A}$ prevalência de obesidade nas mulheres em idade reprodutiva varia de $4 \%$ a $21 \%$ nos países europeus e é de $32 \%$ nos Estados Unidos. ${ }^{4}$

Há evidências de que a obesidade afeta a saúde reprodutiva do binômio materno fetal. Há implicações neonatais em filhos de pais obesos, independentemente do diagnóstico materno de síndrome dos ovários policísticos. ${ }^{5} 0$ prejuízo ocorre tanto para o sexo masculino quanto para o feminino em especial, pois leva à anovulação nas mulheres. Além disso, mesmo as mulheres obesas com ciclos ovulatórios demoram mais para engravidar, comparativamente às não obesas que apresentam ciclos ovulatórios. ${ }^{4}$

Os desfechos obstétricos também sofrem influência negativa da obesidade, uma vez que esta e o sobrepeso elevam os riscos de hipertensão gestacional, préeclâmpsia, diabetes gestacional e parto pré-termo.
Acrescente-se a isso, o aumento na frequência da indicação de parto cesariano e de parto instrumental, além da elevação das taxas de morbimortalidade perinatal e dos riscos, em longo prazo, em relação aos bebês. ${ }^{5}$ Ainda hoje, a literatura é escassa e não oferece dados para concluir se a perda de peso antes da fertilização in vitro (FIV) melhora os resultados reprodutivos, apesar dos muitos benefícios para a saúde em geral e a materno-fetal. ${ }^{6,7}$

Alguns estudos recentes trazem avaliações realizadas com o objetivo de saber se as mudanças no estilo de vida, como dietas e exercícios físicos feitos com o intuito de perder peso, tiveram influência nos resultados da fertilização in vitro. Dois trabalhos randomizados, um com 46 indivíduos e outro com 49, não tiveram poder suficiente para avaliar esse impacto sobre as taxas de gravidez ou de nascido vivo, tampouco sobre os resultados obstétricos.,9 Outros dois estudos, igualmente randomizados, com número mais expressivo de pacientes, um com 577 mulheres e outro com 317 mulheres, mostraram que não existem evidências de que a mudança do estilo de vida com perda de peso antes de efetuar a fertilização in vitro apresente resultados reprodutivos melhores do que realizá-la sem esse cuidado prévio, em especial sobre as taxas de gestação e de nascido vivo., 2,10 Também não encontraram benefícios da perda de peso antes do procedimento em pacientes com síndrome dos ovários policísticos, ao contrário do que a literatura indica.

Além disso, não foram observadas evidências de que o grupo que obteve redução de peso antes do procedimento da fertilização necessitou de menor dose de gonadotrofinas. Igualmente, não ficou comprovada a ocorrência de menores taxas de abortamento. No entanto, houve maiores taxas de gestação espontânea no grupo que passou por mudanças de estilo de vida quando comparado àquele que foi submetido diretamente à fertilização in vitro.,10

Um estudo retrospectivo mostrou que o benefício da perda de peso $(5 \%$ a $10 \%)$ sobre a regularização da menstruação, da ovulação e da taxa de gravidez é obtido somente para as mulheres obesas com idade abaixo de 36 anos. Nas que estão acima dessa faixa etária, o fator idade pesa mais sobre os resultados reprodutivos do que o índice de massa corpórea. ${ }^{11}$ Portanto, adiar a fertilização in vitro em pacientes jovens com o objetivo de perder peso pode ser indicação viável, mas adiar a fertilização em pacientes com mais de 36 anos deve ser desencorajado, uma vez que o fator idade tem influência 
negativa maior sobre os resultados do que o índice de massa corpórea, pelos riscos de diminuição da fertilidade com o avançar da idade.

Os dados de 22.317 ciclos de fertilização in vitro registrados pela Society of Assisted Reproductive Thecnology encontraram tendência de impacto negativo do índice de massa corpórea elevado sobre os resultados de técnicas de reprodução assistida, porém sem significância estatística. ${ }^{12}$

Tendo em vista as controvérsias citadas, o objetivo deste estudo é avaliar a influência do índice de massa corpórea das pacientes submetidas a fertilização in vitro sobre as taxas de gravidez evolutiva e de abortamento em um centro privado de reprodução assistida no Brasil.

\section{MÉTODO}

Trata-se de estudo retrospectivo, no qual foram analisados os resultados de transferências de embriões a fresco em pacientes submetidas a fertilização in vitro no período de janeiro de 2014 a dezembro de 2016. Foram excluídas as mulheres com idade abaixo de 18 anos, as que tiveram transferência de embriões congelados ou com as informações insuficientes no prontuário.

O cálculo do índice de massa corpórea de cada paciente foi embasado em informações de peso e altura descritos no prontuário do doente no dia da captação. Foram considerados três grupos de acordo com o índice em referência, ou seja, grupo A com índice até 24,99; grupo B com índice de 25 a 29,99; grupo C com índice de 30 ou mais. Foi utilizado o parâmetro de obesidade definido pela Organização Mundial da Saúde, baseado no índice de massa corpórea. ${ }^{13}$

O protocolo de estimulação ovariana e a dose de gonadotropina empregada foram escolhidos de acordo com a idade e a reserva ovariana de cada caso. As transferências ocorreram entre o terceiro e o quinto dia de desenvolvimento embrionário, preferencialmente em fase de blastocisto.

O suporte de fase lútea foi realizado com progesterona vaginal micronizada, $600 \mathrm{mg}$ por dia, iniciado no dia seguinte ao da captação dos óvulos, com suspensão na décima segunda semana de gestação.

Na análise, foram avaliados média de idade; número total de pacientes por grupos formados de acordo com o índice de massa corpórea; taxas de gestação, consideradas aquelas com teste HCG positivo (para gravidez por meio da betagonadotrofina coriônica humana); taxas de abortamento e taxas de gestação em curso, consideradas aquelas que obtiveram feto vivo na décima segunda semana de gestação, confirmado por ultrassonografia transvaginal.

Foi realizado teste exato de Fisher para comparar os grupos testados dois a dois, com uso do software Action Stat vs 3 (Estatcamp, 2014), desenvolvido sobre a plataforma $\mathrm{R}$.

\section{RESULTADOS}

No estudo, foram avaliados 567 ciclos de fertilização in vitrocom transferências de embrião a fresco. As médias de idade de cada grupo foram respectivamente 36,2 , 37 e 34,6 anos, sem diferença estatística. A média de índice de massa corpórea foi 23,4 e variou de 15 a 36. Do total dos ciclos analisados, 410 mostraram índice de massa corpórea menor que 25 (grupo A), 135 com índice de 25 a 29,9 (grupo B) e 22 com índice maior ou igual a 30 (grupo C).

As taxas de gestação (tabela) de cada grupo foram, respectivamente, para os grupos $A$, B e C, $54,8 \%$ ( 225 de 410 ), $45,9 \%$ (62 de 135) e 40,9\% (9 de 22 ), e as taxas de abortamento para os respectivos grupos foram $13,8 \%$ (31 de 225 ), 17,8\% (11 de 62 ) e $66,6 \%$ (6 de 9). As taxas de gestação em curso foram de $47,3 \%$ para o grupo $A, 37,7 \%$ para o $B$ e $13,6 \%$ para o C. 0 teste exato de Fisher resultou em diferenças significativas nas taxas de gestação evolutiva entre os grupos $A$ e $C$

Tabela. Resultados dos ciclos de fertilização in vitro de acordo com o índice de massa corpórea

\begin{tabular}{|lccc|}
\hline Variáveis & $\begin{array}{c}\text { Grupo A } \\
\text { IMC }<25 \\
\%(\mathbf{n})\end{array}$ & $\begin{array}{c}\text { Grupo B } \\
\text { IMC 25 a 29,9 } \\
\%(\mathbf{n})\end{array}$ & $\begin{array}{c}\text { Grupo C } \\
\text { IMC } \geq 30 \\
\%(\mathbf{n})\end{array}$ \\
\hline $\begin{array}{l}\text { Taxa de } \\
\text { gestação }\end{array}$ & $54,8(225 / 410)$ & $45,9(62 / 135)$ & $40,9(9 / 22)$ \\
\hline $\begin{array}{l}\text { Taxa de } \\
\text { abortamento }\end{array}$ & $13,8(31 / 225)$ & $17,8(11 / 62)$ & $66,6(6 / 9)$ \\
\hline $\begin{array}{l}\text { Taxa de gestação } \\
\text { evolutiva }\end{array}$ & $47,3(194 / 410)$ & $37,7(51 / 13)$ & $13,6(3 / 22)$ \\
\hline
\end{tabular}

IMC: índice de massa corpórea

"definida como teste betagonadotrofina coriônica humana positivo

${ }^{+} p<0,001$ entre os grupos $A$ e $C$ e $p<0,005$ entre os grupos B e $C$

* definida como feto vivo com doze semanas de gestação

${ }^{5} p<0,001$ entre os grupos $A$ e $C$ e $p<0,005$ entre os grupos $B$ e $C$ 
( $p<0,001)$ e entre os grupos B e C $(p<0,005)$, contudo não apontou diferença estatística entre os grupos $A$ e $B$. Em relação às taxas de abortamento, não houve diferença quando comparados os grupos $A$ e $B(p=$ $0,275)$, porém ocorreu diferença quando comparados os grupos A e C $(p<0,001)$ e B e C $(p<0,005)$.

\section{DISCUSSÃO}

Este estudo, baseado em 567 ciclos de fertilização in vitro com transferência de embrião a fresco, demonstra o efeito negativo dose-dependente do grau de obesidade na taxa de gestação evolutiva, ou seja, revela que quanto maior o índice de massa corpórea pior o resultado reprodutivo. Tal resultado é concordante com a metanálise realizada por Sermondade e colaboradores, em que avaliaram 682.532 ciclos e constatou-se menor taxa de nascidos vivos em pacientes obesas $\left(\geq 30 \mathrm{~kg} / \mathrm{m}^{2}\right)$ submetidas a fertilização in vitro em comparação a pacientes com peso normal (razão de chances 0,85; nível de segurança 95\%; intervalo de confiança 0,84-0,87). ${ }^{14}$

Os números mostram que o efeito negativo sobre a taxa de gestação evolutiva só alcança diferença estatística quando o grupo analisado se encontra em estado de obesidade. Não foi observada diferença entre mulheres com índice de massa corpórea normal e mulheres com sobrepeso. Em uma metanálise, as pacientes com sobrepeso tiveram piores resultados quando comparadas a casos com peso normal (razão de chances 0,94; nível de segurança 95\%; intervalo de confiança 0,91-0,97). ${ }^{14}$ Uma possivel justificativa da ausência de diferença estatística pode ser o tamanho da amostra ou a presença de fatores confundidores não analisados por se tratar de uma investigação retrospectiva.

0 presente estudo mostrou, adicionalmente, maior taxa de abortamento em pacientes obesas $(66,6 \%)$ em comparação a indivíduos com peso normal $(13,8 \%)$ ou a indivíduos com sobrepeso $(17,8 \%)$. Um trabalho em que se avaliaram taxa de abortamento e índice de massa corpórea em mulheres com gestação espontânea atestou haver maior risco de abortamento para as obesas (razão de chances 1,31; nível de segurança 95\%; intervalo de confiança 1,181,46 ) e para aquelas com sobrepeso (razão de chances 1,1 ; nível de segurança $95 \%$; intervalo de confiança 1-1,24), em relação a casos com índice de massa corpórea normal. ${ }^{15}$ Uma metanálise publicada em 2019 mostrou que a obesidade foi também associada a um aumento no risco de abortamento espontâneo recorrente, quando comparada com o peso normal (razão de chances 1,75; nível de segurança 95\%: intervalo de confiança $1,24-2,47 ; p=0,001) .{ }^{16}$

Os mecanismos pelos quais a obesidade afeta a gestação ainda não estão completamente esclarecidos. A obesidade parece promover distúrbio ovulatório secundário à disfunção do eixo hipotálamo-hipófiseovário e redução da fecundidade mesmo em mulheres com ciclos eumenorreicos; afetar a formação do embrião pré-implantacional em razão de desordem da formação do eixo meiótico e da dinâmica mitocondrial; prejudicar a decidualização estromal, levando a uma baixa receptividade endometrial. ${ }^{17}$

Apesar de a fisiopatologia ainda não ser completamente clara, o efeito negativo da obesidade na fertilidade feminina e o aumento de risco materno-fetal durante a gestação são reportados por sociedades internacionais. ${ }^{18-20}$

Dada a importância da obesidade como fator corrigivel, as pacientes são orientadas a perder peso antes de serem submetidas ao tratamento com fertilização in vitro. ${ }^{18-20}$ Embora ainda não esteja comprovado que a redução de peso aumenta a probabilidade de gestação, ela não deve ser observada apenas como um fator de fertilidade, mas como fator prognóstico materno, fetal e pessoal em longo prazo.

Este estudo teve algumas limitações por sua característica de retrospectividade. Não foi avaliada a taxa de nascidos vivos, considerada um dos melhores parâmetros de desfecho em tratamentos de reprodução assistida. Porém, é relatado na literatura que a taxa de abortamento após doze semanas de gestação é menor que $1 \% .{ }^{21}$ Dessa forma, a taxa de gestação evolutiva também seria bom indicador.

\section{CONCLUSÃO}

0 presente estudo mostrou que a obesidade feminina afeta negativamente a taxa de gestação evolutiva em pacientes submetidas a fertilização in vitro, em correlação direta com o grau de afecção do índice de massa corpórea admitido como adequado.

\section{REFERÊNCIAS}

1. Yamakami LYS. Condutas práticas em infertilidade e reprodução assistida: mulher. 1. ${ }^{\text {a }}$ ed., Rio de Janeiro: Elsevier; 2017.

2. Einarsson S, Bergh C, Friberg B, Pinborg A, Klajnbard A, Karlström $P O$ et al. Weight reduction intervention for obese in fertile woman prior to IVF: a randomized controlled trial. 
Hum Reprod. 2017;32(8):1621-30. DOI: 10.1093/humrep/ dex235.

3. Hales CM, Carroll MD, Fryar CD, Ogden CL. Prevalence of obesity among adults and youth: United States, 2015-2016. NCHS Data Brief. 2017 Oct;(288):1-8.

4. Van Oers AM, Groen $H$, Mutsaerts MA, Burggraaff JM, Kuchenbecker WK, Perquin DA et al. Effectiveness of life style intervention in subgroups of obese infertile women: a subgroup analysis of a RCT. Hum Reprod. 2016;31(12):270413. Erratum in: Hum Reprod. 2017;32(2):482. DOI: 10.1093/ humrep/dew252

5. Norman RJ, Mol BWJ. Successful weight loss interventions before in vitro fertilization: fat chance? Fertil Steril. 2018;110(4):581-6. DOI: 10.1016/j.fertnstert.2018.05.029.

6. Homan GF, Davies M, Norman R. The impact of lifestyle factors on reproductive performance in the general population and those undergoing infertility treatment: a review. Hum Reprod Update. 2007;13(3):209-23. DOI: 10.1093/humupd/dml056

7. Fauser BC, Tarlatzis BC, Rebar RW, Legro RS, Balen AH, Lobo $\mathrm{R}$ et al. Consensus on women's health aspects of polycystic ovary syndrome (PCOS): the Amsterdam ESHRE/ ASRM-Sponsored 3rd PCOS Consensus Workshop Group. Fertil Steril. 2012;97(1):28-38.e25. DOI: 10.1016/j. fertnstert.2011.09.024.

8. Moran L, Tsagareli V, Norman R, Noakes M. Diet and IVF pilot study: short-term weight loss improves pregnancy rates in overweight/obese women undertaking IVF. Aust N Z J Obstet Gynaecol. 2011;51(5):455-9. DOI: 10.1111/j.1479828X.2011.01343.x.

9. Sim KA, Dezarnaulds GM, Denyer GS, Skilton MR, Caterson ID. Weight loss improves reproductive outcomes in obese women undergoing fertility treatment: a randomized controlled trial. Clin Obes. 2014;4(2):61-8. DOI: 10.1111/cob.12048.

10. Mutsaerts $M A$, van Oers $A M$, Groen $H$, Burggraaff JM, Kuchenbecker WK, Perquin DA et al. Randomized trial of a lifestyle program in obese infertile women. N Engl J Med. 2016;374(20):1942-53. DOI: 10.1056/NEJMoa1505297.

11. Sneed ML, Uhler ML, Grotjan HE, Rapisarda JJ, Lederer $\mathrm{KJ}$, Beltsos AN. Body mass index: impact on IVF success appear age-related. Hum Reprod. 2008;23(8):1835-9. DOI: 10.1093/ humrep/den 188.

12. Provost MP, Acharya KS, Acharya CR, Yeh JS, Steward RG,
Eaton JL et al. Pregnancy outcomes decline with increasing recipient body mass index: an analysis of 22,317 fresh donor/recipient cycles from the 2008-2010 Society for Assisted Reproductive Technology Clinic Outcome Reporting System registry. Fertil Steril. 2016;105(2):364-8. DOI: 10.1016/j. fertnstert.2015.10.015.

13. WHO Expert Consultation. Appropriate body-mass index for Asian population and its implications for policy and intervention strategies. Lancet. 2004;363(9403):157-63.

14. Sermondade N, Huberlant S, Bourhis-Lefebvre V, Arbo E, Gallot $\mathrm{V}$, Colombani $\mathrm{M}$ et al. Female obesity is negatively associated with live birth rate following IVF: a systematic review and meta-analysis. Hum Reprod Update. 2019;25(4):439-51. DOI: 10.1093/humupd/dmz011.

15. Boots C, Stephenson MD. Does obesity increase the risk of miscarriage in spontaneous conception: a systematic review. Semin Reprod Med. 2011;29(6):507-13. DOI: 10.1055/s0031-1293204.

16. Cavalcante MB, Sarno M, Peixoto AB, Araujo Júnior E, Barini R. Obesity and recurrent miscarriage: $A$ systematic review and meta-analysis. J Obstet Gynaecol Res. 2019;45(1):30-8. DOI: 10.1111/jog.13799

17. Broughton DE, Moley $\mathrm{KH}$. Obesity and female infertility: potential mediators of obesity's impact. Fertil Steril. 2017;107(4):840-7. DOI: 10.1016/j.fertnstert.2017.01.017.

18. Dondorp W, de Wert G, Pennings G, Shenfield F, Devroey P, Tarlatzis B et al. Lifestyle-related factors and access to medically assisted reproduction. ESHRE Task Force on Ethics and Law. Hum Reprod. 2010;25(3):578-83. DOI: 10.1093/ humrep/dep458.

19. National Collaborating Centre for Women's and Children's Health (UK). Fertility: assessment and treatment for people with fertility problems. London: National Institute for Health and Clinical Excellence - Guidance; 2013.

20. Practice Committee of the American Society for Reproductive Medicine. Obesity and reproduction: a committee opinion. Fertil Steril 2015;104:1116-26. DOI: 10.1016/j. fertnstert.2015.08.018

21. Mukherjee S, Velez Edwards DR, Baird DD, Savitz DA, Hartmann KE. Risk of miscarriage among black women and white women in a U.S. Prospective Cohort Study. Am J Epidemiol. 2013;177(11):1271-8. DOI: 10.1093/aje/kws393. 